$11 ; 01.5$

\title{
Экспериментальная модель многолучевого устройства для наблюдения в радиосвете
}

\author{
(C) А.С. Дмитриев, М.М. Петросян, А.И. Рыжов \\ Институт радиотехники и электроники им. В.А. Котельникова РАН, Москва, Россия \\ E-mail: chaos@cplire.ru
}

Поступило в Редакцию 10 марта 2021 г.

В окончательной редакции 10 марта 2021 г.

Принято к публикации 25 марта 2021 г.

Рассматривается возможность создания аналога биологического глаза в радиодиапазоне для наблюдения окружающего пространства в искусственном радиосвете. Как и в биологическом прототипе, в аналоге имеется система чувствительных элементов (сетчатка), фокусирующий элемент типа линзы и совокупность слабонаправленных антенн, на которые осуществляется первичный прием шумоподобного сверхширокополосного излучения. Предложено экспериментальное устройство рассматриваемого типа и показана работоспособность предлагаемого технического решения.

Ключевые слова: искусственное радиоосвещение, сверхширокополосные сигналы, динамический хаос, приемники радиосвета, многолучевые средства наблюдения.

DOI: 10.21883/PJTF.2021.12.51066.18762

Системы наблюдения за окружающим пространством с применением микроволнового излучения в последнее время привлекают большое внимание в связи с задачами навигации в пространстве роботов различного назначения, мобильных беспилотных средств, контроля за передвижением объектов в ограниченном пространстве и т.д. Как правило, для этих целей используются активные системы, включающие в себя передатчик, подсвечивающий объект и приемник (или, как вариант, разнесенную систему приемников).

При этом активно используются элементы существующих узкополосных средств связи, например WiFi $[1,2]$, сверхширокополосные средства с импульсными сигналами [3], системы, использующие шумовые широкополосные сигналы [4].

Использование искусственного радиоосвещения [5,6] представляет собой альтернативную концепцию, в которой источники (лампы радиосвета) освещают сразу всю наблюдаемую зону, а системы наблюдения принципиально являются пассивными и производят наблюдение за окружающей обстановкой, не излучая сигналов. Это приводит к двум важным следствиям. Во-первых, такую систему нельзя обнаружить по собственному излучению, и тем самым стороннему наблюдателю сложно понять, ведется ли в этой зоне за какими-то объектами наблюдение. Во-вторых, наблюдение может одновременно вестись по многим направлениям без сканирования, т.е. с применением многолучевых систем, что увеличивает скорость обзора пространства пропорционально количеству используемых лучей.

Одновременное наблюдение за значительной частью окружающего пространства широко используется в природе с помощью глаз животными в видимом диапазоне электромагнитного спектра (большинство живот- ных) [7], инфракрасном диапазоне (змеи) [8] и ультрафиолетовом диапазоне (бабочки) [9].

В животном мире используются глаза разной структуры, но большинство типов глаз включает в себя линзовую систему и сетчатку, состоящую из множества чувствительных элементов: палочек и колбочек. Число этих чувствительных элементов колеблется от нескольких десятков у простейших животных до нескольких миллионов у высших животных.

Если следовать по пути имитации глаза для построения системы наблюдения, прежде всего следует создать чувствительный элемент - аналог колбочки. Такой элемент был разработан на основе логарифмического детектора [10]. Он обеспечивает прием и накопление энергии шумоподобного микроволнового излучения, подобно тому как это делается в радиометрах. При использовании этого элемента совместно с направленной приемной системой была показана возможность получения изображений в радиосвете. Это достигалось путем механического сканирования лучом высоконаправленной приемной системы в пространстве [11]. Предложенное решение позволило продемонстрировать принципиальную возможность наблюдения окружающей обстановки в радиосвете, но имело существенные ограничения для практических приложений, в частности связанные с низкой скоростью сканирования.

$\mathrm{B}$ настоящей работе описываются эксперименты с макетом устройства наблюдения за окружающим пространством, имитирующего глаз с хрусталиком и сетчаткой, в котором не используется механическое сканирование для получения пространственного разрешения. Макет устройства включает в себя аналог сетчатки, состоящий из $N$ чувствительных элементов, линзовый элемент аналог хрусталика, обеспечивающий фокусировку сигна- 

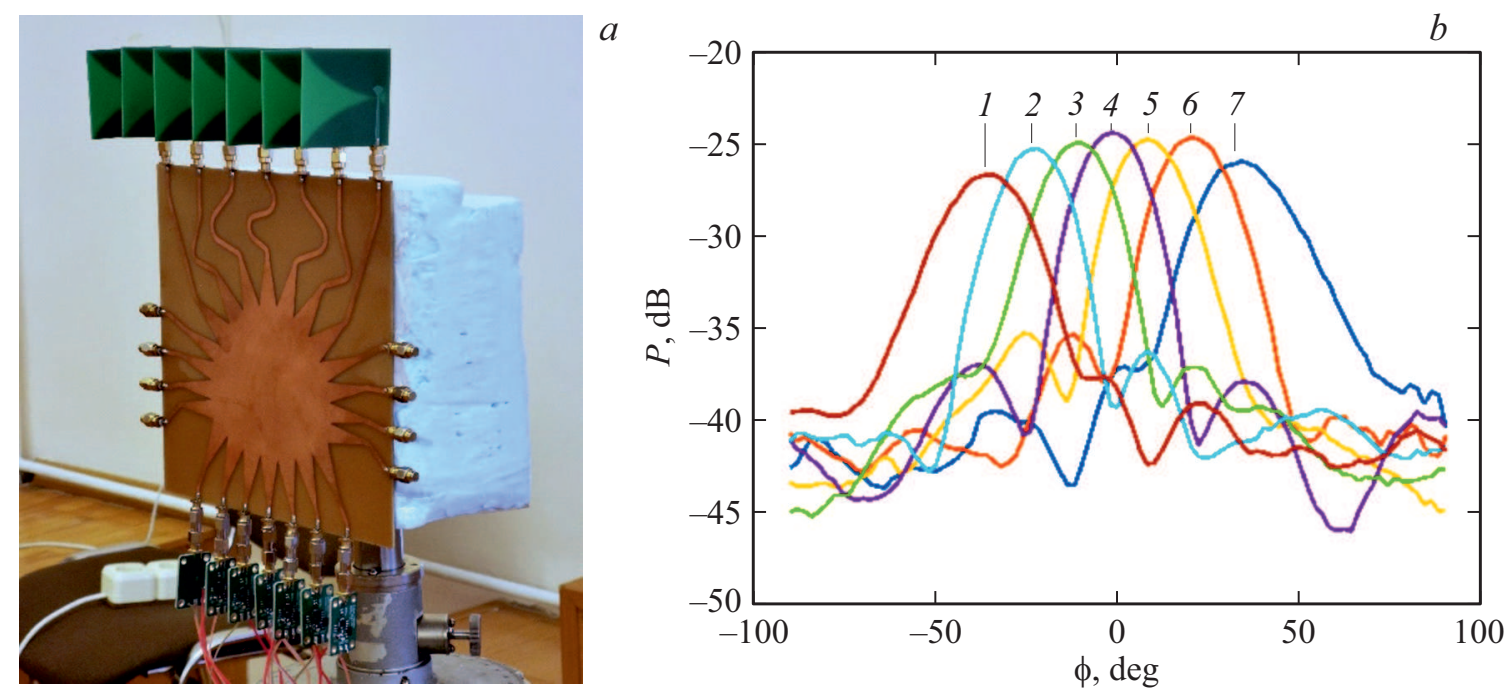

Рис. 1. Экспериментальный макет устройства наблюдения в микроволновом диапазоне. $a-$ общий вид; $b-$ диаграммы направленности лучей $1-7$ устройства.

лов, поступающих с определенного направления, и собственно приемные элементы в виде слабонаправленных антенн на входе линзы.

Разработанный макет предназначен для частотного диапазона 3-5 GHz. В качестве источников излучения использовались лампы радиосвета, в которых сверхширокополосный шумоподобный сигнал представляет собой динамический хаос микроволнового диапазона $[5,6]$.

Аналог хрусталика глаза был реализован в виде радиолинзы. В радиодиапазоне известно несколько типов линз [12], которые могут быть использованы для концентрации приходящего излучения, в частности линза Люнеберга, идея которой очень напоминает функцию хрусталика. С ее помощью можно образовать устройство, похожее по принципу действия на глаз того типа, который характерен для млекопитающих.

Однако в сантиметровом диапазоне это довольно габаритное устройство. Поэтому для исследований в работе был выбран вариант построения приемной системы на основе линзы Ротмана $[12,13]$.

На входах линзы размещались приемные элементы на основе антенн Вивальди (рис. 1, $a$ ). Число приемных элементов и формируемых лучей равно семи (рис. $1, b)$, сектор обзора $60^{\circ}$, частотный диапазон $3-5 \mathrm{GHz}$ (соответствует диапазону источников радиосвета).

Топология линзы для заданных параметров была рассчитана в программном комплексе [14].

Наблюдение за предметами в окружающем пространстве при освещении радиосветом производилось следующим образом. На одной из стен помещения размещались две лампы радиосвета мощностью $2 \mathrm{~mW}$ каждая [5,6] таким образом, чтобы осуществлялось равномерное освещение помещения и прямые лучи от ламп не попадали в приемные элементы устройства. Наблюдаемый предмет располагался перед устройством на расстоянии от 1 до $3 \mathrm{~m}$ от него. Превышение плотности мощности искусственного радиоосвещения над фоновым значением составляло 20-30 dB. Предполагалось, что отраженные от предмета лучи ламп радиосвета будут попадать в апертуру „глаза“ и давать отклик на одном или нескольких выходах системы. Наличие этих сигналов, сильно отличающихся по уровню от сигналов, принятых с направлений, где отражение от предмета отсутствует, будет свидетельствовать о том, в что в этом направлении находится отражающий предмет.

Были проведены две группы экспериментов, в которых в качестве отражающих предметов использовались рассеивающие металлические поверхности. В первом случае это был квадрат размером примерно $0.7 \times 0.7 \mathrm{~m}$, расположенный на расстоянии $2 \mathrm{~m}$ от наблюдательного устройства и покрытый негладкой фольгой с характерными размерами неоднородности $3-4 \mathrm{~cm}$, обеспечивающей диффузное отражение (рис. 2,a). Во втором случае в качестве наблюдаемых предметов использовались два разнесенных примерно на $1.5 \mathrm{~m}$ квадрата с негладкой фольгой размером $0.5 \times 0.5 \mathrm{~m}$ (рис. $3, a$ ).

Результаты экспериментов представлены на рис. 2, $b$ и $3, b$. По оси абсцисс отложены значения углов в горизонтальной плоскости. Квадратами обозначены направления максимумов диаграммы направленности каждого из семи лучей и уровни приходящей с этих направлений мощности.

На рис. 2, $b$ отраженные от единственного объекта (рис. 2,a) лучи попадают в основные лепестки трех диаграмм направленности приемной системы (2, 3 и 4), в остальные четыре диаграммы направленности сигнал попадает только через боковые лепестки, и уровень итогового сигнала с этих направлений значительно ниже. Уровень мощности сигналов, принимаемых со всех семи направлений, позволяет четко идентифицировать 

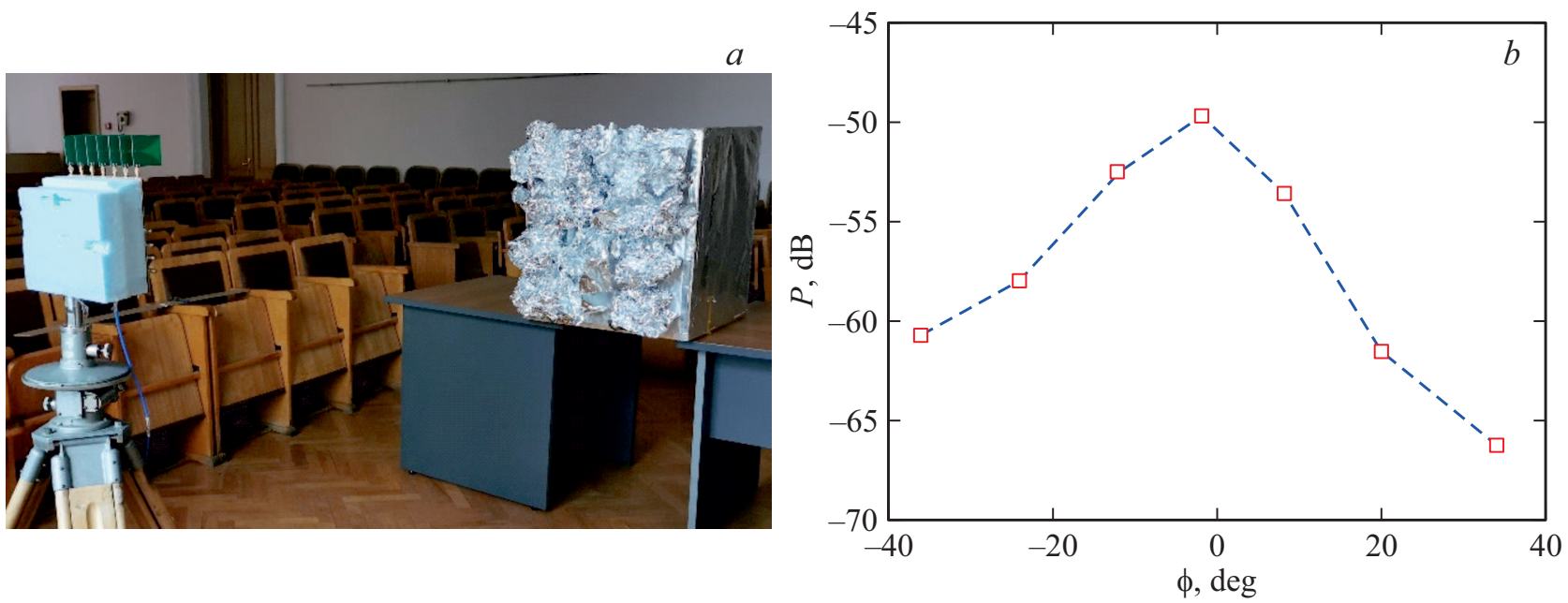

Рис. 2. Эксперименты с одним отражающим объектом. $a-$ взаимное расположение приемного устройства и объекта; $b-$ значения принятой мощности сигнала для каждого из семи лучей.
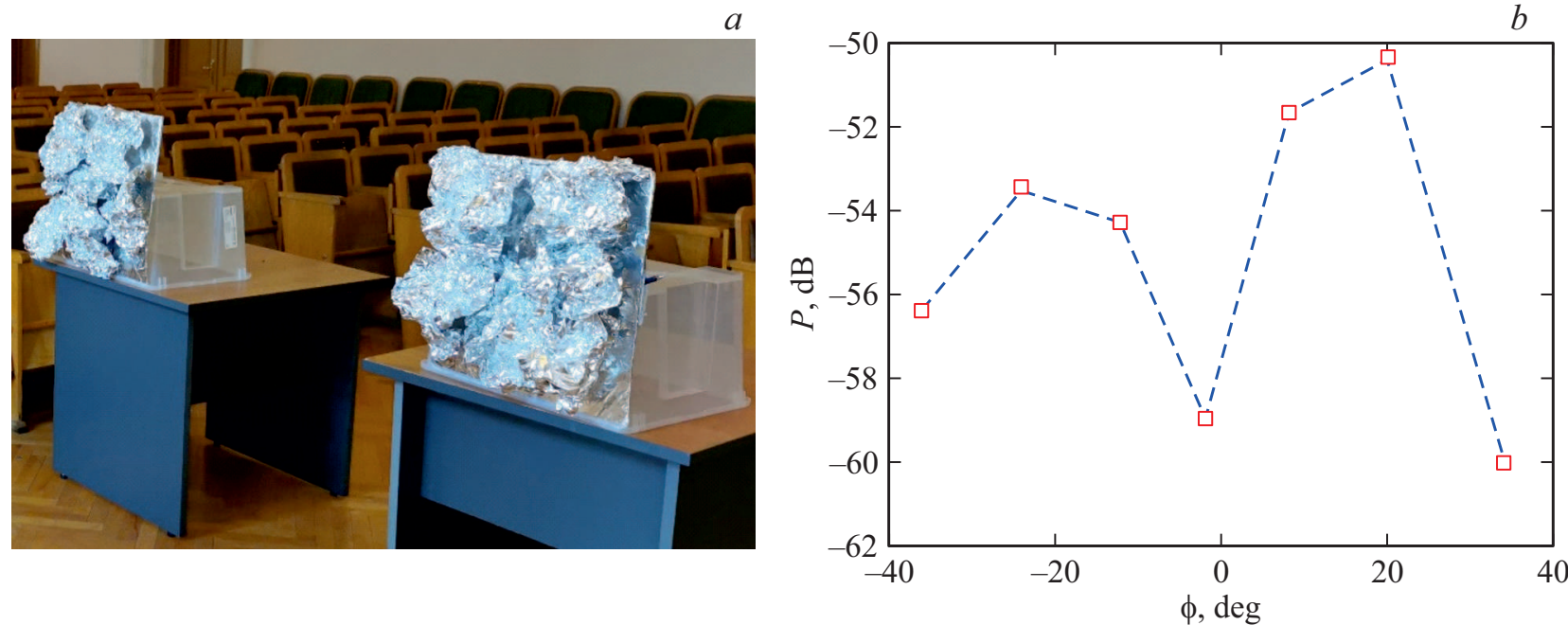

Рис. 3. Эксперименты с двумя отражающими объектами. $a$ - общий вид объектов; $b-$ значения принятой мощности сигнала для каждого из семи лучей.

наличие предмета (ему соответствуют три центральные точки с максимальными значениями сигнала), а также грубо оценить его размер: угловое расстояние между третьей и пятой точками составляет примерно $20^{\circ}$, что при расстоянии $2 \mathrm{~m}$ от „глаза“ до предмета соответствует линейному размеру $\sim 0.7 \mathrm{~m}$.

На рис. 3, $b$ представлена картина, наблюдаемая при наличии в поле зрения приемного устройства двух рассеивающих предметов меньшего размера (рис. 3,a). В этом случае четко идентифицируется наличие обоих объектов: вторая и третья точки соответствуют первому объекту, пятая и шестая точки - второму объекту (рис. $3, b$ ). Об их размерах можно лишь сказать, что они меньше, чем в первом случае.

Таким образом, проведенные исследования показали возможность наблюдения объектов в радиосвете с помощью многолучевых систем, подобных по своим свойствам глазам биологических объектов с малым числом чувствительных элементов. Отметим, что плотность мощности радиоосвещения в экспериментах была примерно на три порядка ниже, чем требуется при типичном уровне освещения в видимом диапазоне света.

\section{Финансирование работы}

Работа выполнена по государственному заданию Института радиотехники и электроники им. В.А. Котельникова РАН.

\section{Конфликт интересов}

Авторы заявляют, что у них нет конфликта интересов. 


\section{Список литературы}

[1] A. Muralidharan, Y. Mostofi, in 2016 IEEE Int. Conf. on acoustics, speech and signal processing (ICASSP) (Shanghai, China, 2016), p. 6385. DOI: 10.1109/ICASSP.2016.7472906

[2] R.S. Campos, L. Lovisolo, M.L. de Campos, Expert Syst. Appl., 41 (14), 6211 (2014).

DOI: 10.1016/j.eswa.2014.04.011

[3] P. Galajda, A. Galajdova, S. Slovak, M. Pecovsky, M. Drutarovsky, M. Sukop, I. BA Samaneh, Int. J. Adv. Robot. Syst., 15 (4), 1 (2018). DOI: 10.1177/1729881418795767

[4] С.И. Ивашов, А.С. Бугаев, Радиотехника и электроника, 58 (9), 935 (2013). DOI: 10.7868/S0033849413090052

[5] А.С. Дмитриев, Е.В. Ефремова, М.Ю. Герасимов, В.В. Ицков, Радиотехника и электроника, 61 (11), 1073 (2016). DOI: $10.7868 / \mathrm{S} 0033849416110024$

[6] А.С. Дмитриев, Е.В. Ефремова, Письма в ЖТФ, 42 (24), 49 (2016). DOI: 10.21883/pjtf.2016.24.44078.16439

[7] К.Ю.М. Смит, Биология сенсорных систем (БИНОМ, М., 2013).

[8] A.B. Sichert, P. Friedel, J.L. van Hemmen, Phys. Rev. Lett., 97 (6), 068105 (2006). DOI: 10.1103/PhysRevLett.97.068105

[9] R.H. Douglas, G. Jeffery, Proc. Roy. Soc. B, 281 (1780), 20132995 (2014). DOI: 10.1098/rspb.2013.2995

[10] Ю.В. Гуляев, А.С. Дмитриев, В.В. Ицков, М.М. Петросян, А.И. Рыжов, А.В. Уваров, Радиотехника и электроника, 63 (9), 947 (2018). DOI: 10.1134/S0033849418090085

[11] А.С. Дмитриев, В.В. Ицков, М.М. Петросян, М.Г. Попов, А.И. Рыжов, Радиотехника и электроника, 64 (9), 916 (2019). DOI: 10.1134/S0033849419080047

[12] С.Е. Банков, Интегральная СВЧ-оптика (Физматлит, М., 2018), c. 534

[13] A. Rabinovich, N. Alexandrov, Antenna a rrays and automotive applications (Springer, N.Y., 2013), p. 180.

[14] M. Pokorny, Rotman lens design with HFSS [Электронный pecypc]. Режим доступа: https://www.mathworks.com/ matlabcentral/fileexchange/50490-rotman-lens-design-withhfss-link 